\title{
Variations in Clinical PET/CT Operations: Results of an International Survey of Active PET/CT Users
}

\author{
Thomas Beyer ${ }^{1,2}$, Johannes Czernin ${ }^{3}$, and Lutz S. Freudenberg ${ }^{1}$ \\ ${ }^{1}$ Department of Nuclear Medicine, University Hospital Essen, Essen, Germany; ${ }^{2}$ cmi-experts GmbH, Zürich, Switzerland; and \\ ${ }^{3}$ Department of Molecular and Medical Pharmacology, David Geffen School of Medicine at UCLA, Los Angeles, California
}

This study gathered information about clinical PET/CT operations worldwide to help guide discussions on the use and standardization of clinical PET/CT. Methods: A Web-based survey of PET/CT users was initiated in November 2009 through e-mail advertising using Academy of Molecular Imaging databases. Recipients were asked 58 questions related to demographics (e.g., location, number of PET/CT systems, and staffing), PET/CT operations and use, and variations in ${ }^{18} \mathrm{~F}-$ FDG oncology imaging protocols. Results: The responders were from centers in the Americas (71\%), Europe (22\%), AsiaPacific (6\%), and Middle East (1\%), with most responding sites representing public health care institutions (60\%). PET/CT systems were most frequently installed in nuclear medicine departments $(59 \%)$. Of the sites operating a PET/CT system, $16 \%$ had $10 \mathrm{y}$ or more of stand-alone PET experience. About $40 \%$ of all sites operated at least 2 PET/CT systems. PET/CT was most frequently used for applications in torso or whole-body oncology $(87 \%)$, radiation therapy planning $(4 \%)$, cardiology $(4 \%)$, and neurology $(5 \%)$. The average interval of fasting before an ${ }^{18}$ F-FDG PET/CT examination was $7 \pm 3 \mathrm{~h}$ (range, 4-12 h). Blood glucose levels were measured at $99 \%$ of sites, but acceptable maximal glucose levels varied substantially (an upper limit of $200 \mathrm{mg} / \mathrm{dL}$ was applied at $>50 \%$ of the institutions). A weight-based radioactivity dose injection was performed at $44 \%$ of sites. The mean ${ }^{18} \mathrm{~F}-\mathrm{FDG}$ activity injected was $390 \mathrm{MBq}$ (range, 110-585 MBq) for 3-dimensional PET of a $75-\mathrm{kg}$ patient. The mean uptake time was $64 \pm 14 \mathrm{~min}$ (range, 20-90 min). Split protocols involving patient repositioning and adapted imaging parameters were used at $51 \%$ of sites. Only $41 \%$ used patient positioning aids. Intravenous or oral CT contrast material was used at $52 \%$ of sites in up to $25 \%$ of patients. Most sites (90\%) measured maximum standardized uptake value as an index of tissue glucose use. Only $62 \%$ of sites provided a fully integrated PET/CT report. Conclusion: An international survey among clinical PET/CT users revealed significant variations in standard ${ }^{18} \mathrm{~F}-\mathrm{FDG}$ PET/CT protocols. This finding illustrates the need for continuous training and ongoing standardization in an effort to optimize PET/CT in oncology.

\footnotetext{
Received May 28, 2010; revision accepted Jul. 21, 2010.

For correspondence or reprints contact: Lutz S. Freudenberg, Department of Nuclear Medicine, University Hospital Essen, Hufelandstrasse 55, D-45122 Essen, Germany.

E-mail: lutz.freudenberg@uni-duisburg-essen.de

Guest Editor: Barry Siegel, Mallinckrodt Institute of Radiology

COPYRIGHT @ 2010 by the Society of Nuclear Medicine, Inc.
}

Key Words: PET/CT; clinical operations; imaging protocols

J Nucl Med 2011; 52:303-310

DOI: 10.2967/jnumed.110.079624

C ombined PET/CT systems were first proposed in 1998 and were made commercially available in 2001 (1). Since then, more than 5,000 combined PET/CT tomographs have been installed worldwide, and PET/CT is today considered one of the fastest-growing imaging modalities. The introduction of PET/CT was met with early skepticism, which prevails today in some countries or medical disciplines despite growing evidence of the diagnostic superiority of combined PET/CT over stand-alone PET and CT systems (2).

Nonetheless, the combination of 2 complementary imaging modalities into a single imaging device creates several operational and logistic challenges that, if neglected, bear the risk of poor-quality examinations, suboptimal diagnostic results, the need to repeat examinations, limited comparability among studies performed at different institutions, and, consequently, poor therapy decisions. Therefore, procedure guidelines for clinical and research studies have been proposed (3-6). In addition, recommendations on appropriate training are under consideration for both CT and PET and should aid in establishing appropriately trained PET/CT professionals as well (7).

In 2009, Graham et al. presented a survey on variations in the use of combined PET/CT in 15 U.S.-based academic imaging centers $(8)$. They reported considerable variability in PET/CT protocols that would limit the validity of PET data from multicenter trials.

This study undertook a worldwide survey of public and private imaging centers to assess current PET/CT protocols. The data should be useful for reinforcing standardization attempts and for implementing such protocols in research and in clinical practice.

\section{MATERIALS AND METHODS}

In October 2009, we conceptualized an international survey to collect operations and protocol data from users of clinical PET/CT systems. The idea for this data collection came from a national 
survey by the Imaging Response Assessment Team funded by the National Cancer Institute, reported in this issue of The Journal of Nuclear Medicine (8).

Despite the known drawbacks of Web-based surveys, such as a lower response rate than for other survey modes $(9,10)$, we decided to benefit from the easy, rapid, and widespread distribution of Web-based questionnaires. Furthermore, Web-based surveys offer logistic advantages such as fast response collection and low costs $(9,11,12)$.

We prepared our survey in line with recent recommendations on the structure of surveys-recommendations that, in theory, help maximize response rates. These recommendations included a personal introductory statement, the offer to make the results public, simple headers and textual representation of response categories, and a foreseeable deadline with multiple reminders (13).

The electronic survey had 58 questions (shown in the supplemental materials, available online only at http://jnm.snmjournals. org), which could be answered in less than $25 \mathrm{~min}$. The questions were developed by imaging experts with at least 8 y of clinical experience in combined PET/CT. The questions covered 3 categories: demographics (e.g., PET/CT location and number of systems), PET/CT operations (e.g., throughput, staffing, and major indications), and ${ }^{18} \mathrm{~F}-\mathrm{FDG}$ PET/CT oncology imaging protocols.

The survey, in English, was composed using a Web-based platform (SurveyMonkey.com). A link to the survey was e-mailed in November 2009 to the complete list of members of the Academy of Molecular Imaging (913 registered users). We had also requested support from the European Association of Nuclear Medicine (EANM) and the European Society of Radiology for wider dissemination of the survey. In view of the lack of a timely endorsement of this survey by either association, responses were gathered only from the direct e-mailing to the Academy of Molecular Imaging. Three reminders were e-mailed to all nonresponding recipients between December 2009 and April 2010.

Responses were collected anonymously and tabulated for each question. We report total responses per category; arithmetic mean, minimum, and maximum values (when applicable); and individual free-text responses. We review selected responses with respect to the recommendations from existing guidelines on the use of ${ }^{18}$ F-FDG PET/CT in oncology (4-6).

\section{RESULTS}

We collected 128 responses between November 11, 2009, and April 28, 2010, for an overall response rate of $14 \%$.

\section{Demographics}

The responders included department directors (51\%), physicians $(31 \%)$, technologists $(14 \%)$, physicists $(8 \%)$, and others $(6 \%)$. The responding sites were in North or South America (71\%), Europe (22\%), Asia-Pacific (6\%), and the Middle East (1\%). Sixty percent of all PET/CT operations were public, $33 \%$ were private, and $7 \%$ were public-private partnerships. Most sites (63\%) operated 1 PET/CT system, and $18 \%$ of sites operated 2 PET/CT systems (Table 1). Three quarters of all PET/CT operations served a population of 1 million people or fewer.

At $84 \%$ of sites, all PET/CT systems were installed within the same department: nuclear medicine (59\%),
TABLE 1

Number of PET/CT Systems Operated at a Single Location

\begin{tabular}{lc}
\hline $\begin{array}{l}\text { Number } \\
\text { of PET/CT systems }\end{array}$ & $\begin{array}{c}\text { Percentage of locations with this } \\
\text { number of systems }(n=104)\end{array}$ \\
\hline 1 & 63 \\
2 & 18 \\
3 & 7 \\
4 & 5 \\
5 & 4 \\
6 & 3 \\
\hline 7 & 1 \\
\hline
\end{tabular}

radiology $(18 \%)$, or another department $(23 \%)$. The other departments most frequently listed were molecular imaging, oncology, or radiation oncology.

Fifty-nine percent of sites had previous experience with PET-only systems, which had been in use for $1-5$ y (53\%), $6-10$ y $(20 \%)$, or more than 10 y $(27 \%)$. The other $41 \%$ of sites had experience only with hybrid PET/CT systems.

\section{PET/CT Operations}

The PET/CT systems were installed by GE Healthcare, Philips Healthcare, or Siemens Healthcare. Of the sites that added a second or third PET/CT system, most sites (76\%) chose the same vendor as for the first PET/CT system.

The source of the PET tracers was an in-house cyclotron for $33 \%$ of sites, an in-house generator for $9 \%$ of sites, and an external provider for $81 \%$ of sites, suggesting multiple sources of radiotracers per site.

Of the sites that purchased commercial PET radiopharmaceuticals, $67 \%$ depended on a single supplier, $26 \%$ on 2 suppliers, and $7 \%$ on 3 suppliers. In general, ${ }^{18} \mathrm{~F}$ labeled compounds were the most widely used radiotracers (Table 2).

When several PET/CT systems were available at a site, the first system was most frequently used for torso and total-body examinations for oncology, and the additional

TABLE 2

Percentage of Patient PET/CT Examinations Performed Using a Particular Tracer

\begin{tabular}{|c|c|}
\hline PET radiotracer & $\begin{array}{c}\text { Percentage of patient } \\
\text { examinations using this tracer }\end{array}$ \\
\hline${ }^{18} \mathrm{~F}-\mathrm{FDG}$ & 100.0 \\
\hline${ }^{18}$ F-fluoride & 29.5 \\
\hline${ }^{11} \mathrm{C}$ - and ${ }^{18} \mathrm{~F}$-choline & 23.9 \\
\hline $\begin{array}{l}{ }^{11} \mathrm{C}-\text { and }{ }^{18} \mathrm{~F}-3,4- \\
\text { dihydroxyphenylalanine }\end{array}$ & 17.0 \\
\hline${ }^{18}$ F-fluoro-ethyl-L-tyrosine & 13.6 \\
\hline${ }^{68} \mathrm{Ga}-\mathrm{DOTATOC}$ & 9.1 \\
\hline${ }^{11} \mathrm{C}$ - and ${ }^{18} \mathrm{~F}$-acetate & 5.7 \\
\hline $124 \mid$ & 4.5 \\
\hline Other & 34.1 \\
\hline Data are averaged across & es. \\
\hline
\end{tabular}


systems were used for radiation therapy planning, cardiology applications, and neurology applications (Supplemental Fig. 1). The 3 most frequent indications for PET/CT were lymphoma, lung cancer, and breast cancer. Cardiac and neurologic PET/CT examinations were most frequently ordered for myocardial viability and dementia assessments, respectively (Table 3). Most patients got a PET/CT appointment within $1 \mathrm{wk}$, independent of the indication (Table 4).

The sites used an average of 4.9 full-time-employee technologists, 1.3 full-time-employee physicists, 2.5 fulltime-employee radiologists, and 2.5 full-time-employee nuclear medicine experts.

\section{F-FDG PET/CT Protocols}

Patient Preparation. The average fasting period for oncology imaging using ${ }^{18} \mathrm{~F}-\mathrm{FDG} \mathrm{PET} / \mathrm{CT}$ varied among users: $0-4 \mathrm{~h}(27 \%), 5-6 \mathrm{~h}(51 \%), 7-8 \mathrm{~h}(9 \%)$, or more than $8 \mathrm{~h}(13 \%)$. About one third of the responders reported additional dietary requirements for oncology studies, such as no caffeine, a low-carbohydrate diet, or nothing taken orally.

Blood sugar levels were checked by almost all sites (99\%) before ${ }^{18}$ F-FDG injection. However, blood sugar cutoff levels varied widely-from 150 to $250 \mathrm{mg} / \mathrm{dL}$ - with

TABLE 3

Most Frequent Indications for PET/CT in Oncology, Cardiology, and Neurology

\begin{tabular}{|c|c|}
\hline Indication & $\begin{array}{l}\text { Percentage of systems } \\
\text { used for this indication }\end{array}$ \\
\hline Oncology & $\begin{array}{l}\text { Oncology systems } \\
\qquad(n=298)\end{array}$ \\
\hline Lung cancer & 29.9 \\
\hline Lymphoma & 24.5 \\
\hline Breast cancer & 12.4 \\
\hline Colorectal cancer & 11.7 \\
\hline Head and neck cancer & 8.1 \\
\hline Melanoma & 2.7 \\
\hline Gastrointestinal stroma tumor & 1.0 \\
\hline Myeloma & 1.0 \\
\hline Cancer of unknown primary & 1.0 \\
\hline Esophageal cancer & 1.0 \\
\hline Prostate cancer & 0.7 \\
\hline Other tumors & 6.0 \\
\hline Cardiology & $\begin{array}{l}\text { Cardiology systems } \\
\quad(n=62)\end{array}$ \\
\hline Viability & 59.7 \\
\hline Ischemia & 37.1 \\
\hline Research & 1.6 \\
\hline Aortic inflammation & 1.6 \\
\hline Neurology & $\begin{array}{l}\text { Neurology systems } \\
\qquad(n=174)\end{array}$ \\
\hline Dementia & 35.1 \\
\hline Brain tumor & 24.1 \\
\hline Epilepsy & 17.8 \\
\hline Alzheimer disease & 9.8 \\
\hline Seizure & 5.2 \\
\hline Parkinson disease & 5.2 \\
\hline Other & 2.9 \\
\hline
\end{tabular}

TABLE 4

Average Postreferral Waiting Time for a Scheduled PET/CT Examination

\begin{tabular}{lccc}
\hline & \multicolumn{2}{c}{ Percentage of sites with this waiting time } \\
\cline { 2 - 4 } $\begin{array}{l}\text { Waiting } \\
\text { time }(\mathrm{d})\end{array}$ & $\begin{array}{c}\text { Oncology } \\
(n=99)\end{array}$ & $\begin{array}{c}\text { Cardiology } \\
(n=34)\end{array}$ & $\begin{array}{c}\text { Neurology } \\
(n=72)\end{array}$ \\
\hline 1 & 26 & 32 & 26 \\
2 & 28 & 29 & 35 \\
$3-7$ & 30 & 29 & 26 \\
$8-14$ & 9 & 6 & 8 \\
$>14$ & 6 & 3 & 4 \\
\hline
\end{tabular}

most sites (52\%) accepting a cutoff of $200 \mathrm{mg} / \mathrm{dL}$ and $7 \%$ of sites reporting no cutoff level (Supplemental Fig. 2).

Radiopharmaceutical. ${ }^{18} \mathrm{~F}-\mathrm{FDG}$ administration was based on patient weight at $44 \%$ of sites. The average ${ }^{18} \mathrm{~F}-\mathrm{FDG}$ dose for oncologic studies was $5.2 \mathrm{MBq}$ of ${ }^{18} \mathrm{~F}-\mathrm{FDG}$ per kilogram of body weight, and the range was $1.5-7.8 \mathrm{MBq} /$ $\mathrm{kg}$. This average dose translates into an average ${ }^{18} \mathrm{~F}-\mathrm{FDG}$ activity of $390 \mathrm{MBq}$ (range, 113-585 MBq) injected into a $75-\mathrm{kg}$ patient. Sites that did not use a weight-based activity injection $(56 \%)$ had established maximum ${ }^{18} \mathrm{~F}-\mathrm{FDG}$ activity limits averaged across sites: $524 \mathrm{MBq}$ (range, 370-666 $\mathrm{MBq}$ ) for 2-dimensional (2D) PET and $465 \mathrm{MBq}$ (range, 200-740 MBq) for 3-dimensional (3D) PET. Uptake times of $45 \mathrm{~min}$ or less were the standard at $8 \%$ of all sites. Only half the sites $(49 \%)$ indicated an uptake period of around $60 \mathrm{~min}$ (range, 55-70 min), and 20\% of sites allowed for an uptake period of more than 75 min for oncology protocols.

Image Acquisition. The upper and lower limits for a torso PET/CT protocol were generally defined as the neck and thighs, respectively, but individual descriptions of anatomic landmarks varied significantly among sites (Fig. 1).

In addition to a standard torso imaging protocol involving a topogram, a CT scan, and an emission acquisition, a split protocol is an option (14). Split protocols allow for division of a predefined coaxial imaging range (e.g., torso) into 2 or more ranges as defined by the number of PET bed positions, with the individual CT and PET acquisition parameters adjusted to the imaging range. At $51 \%$ of sites, split protocols that include patient repositioning were used as part of

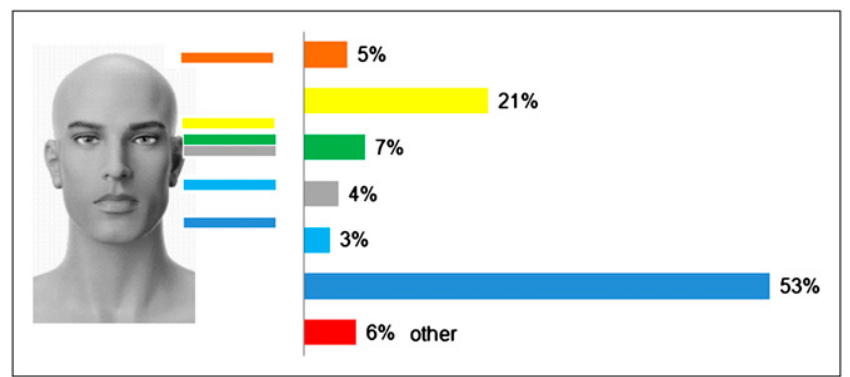

FIGURE 1. Definitions of craniocaudal anatomic limits of torsoPET/CT ranges as percentage of sites. The various anatomic levels are color-coded on topogram of head and neck. 
TABLE 5

Typical PET/CT Indications for Split Protocols

\begin{tabular}{lc}
\hline \multicolumn{1}{c}{ Indication } & $\begin{array}{c}\text { Percentage of sites eligible to } \\
\text { perform split protocol for this } \\
\text { indication }(n=77)\end{array}$ \\
\hline Head and neck cancer & 55.8 \\
\hline Melanoma & 10.4 \\
\hline Thyroid cancer & 7.8 \\
\hline Radiotherapy planning & 6.5 \\
Lung cancer & 3.9 \\
\hline Breast cancer & 2.6 \\
CT contrast study & 2.6 \\
Brain tumors & 2.6 \\
\hline Other tumors & 7.8 \\
\hline
\end{tabular}

routine oncology imaging procedures (Table 5), but only $41 \%$ of all sites used dedicated patient positioning devices, such as a vacuum mattress, thermoplastic masks, or straps.

Use of a dedicated low-dose CT acquisition for attenuation correction of PET/CT images was reported by $73 \%$ of all sites. The low-dose CT scans were typically acquired using a tube voltage of $100-140 \mathrm{kVp}$, a tube current product of at least $10 \mathrm{mAs}$, and an axial slice width of $5 \mathrm{~mm}$.

Thirty-six percent of sites reported using CT contrast material in more than half their patients, and $52 \%$ of sites reported using CT contrast material in less than a quarter of their patients. Table 6 summarizes the frequency of intravenous and oral contrast use in PET/CT studies. More users were inclined to use oral than intravenous contrast material. Oral contrast material was barium-based (39\% sites), iodinebased (35\%), water-based (22\%), or of another type (4\%). In $62 \%$ of contrast-enhanced protocols, the CT images of the PET/CT scan were also used for CT-based attenuation correction, whereas in $38 \%$ of contrast-enhanced protocols, a separate low-dose CT scan was acquired for attenuation correction.

A multibed emission acquisition follows the CT acquisition. The average emission acquisition time per bed position was $3.2 \mathrm{~min}$, with a minimum and maximum of $1 \mathrm{~min}$ and $10 \mathrm{~min}$, respectively. Emission acquisition times were independent of patient weight at two thirds of sites. When adjusted to patient weight, the average emission acquisition time per bed position was 3.1 min for a standard $75-\mathrm{kg}$ patient, with a minimum and maximum of $0.5 \mathrm{~min}$ and 10 min, respectively.

Interpretation and Reporting. PET images were reconstructed in 2 dimensions (20\%) or 3 dimensions (80\%) after CT-based attenuation correction; $48 \%$ of sites used standard image reconstruction on $128 \times 128$ matrices, whereas $52 \%$ used alternative protocols with adapted reconstruction parameters.

Images were reviewed and reported in one of several ways: on the console (58\%), using PACS only (16\%), using open-source software (9\%), or using another method (17\%). These other methods included PACS and a third-party plugin, third-party software, and remote workstations.

Clinical PET/CT studies are interpreted by nuclear medicine physicians at $60 \%$ of sites and by double-trained physicians at $37 \%$ of sites (Supplemental Fig. 3). Several alternative reporting scenarios were listed also, including joint readings for selected indications (e.g., myeloma), interpretation of contrast-enhanced CT (if performed) by radiologists, measurement of standardized uptake values (SUVs) by radiologists, and double reading.

PET/CT interpretations are provided as fully integrated reports by $62 \%$ of sites, as an integrated report with separate paragraphs on PET and CT findings by $20 \%$ of sites, and as separate reports by $17 \%$ of sites.

Ninety percent of sites indicated that SUVs are generally measured and included in the PET/CT reports, and $10 \%$ of sites do not report SUVs. Of the sites that do include SUV, $91 \%$ report maximum SUV, $12 \%$ report mean SUV, $1 \%$ report SUV corrected for lean body mass, and $1 \%$ report SUV corrected for total lesion glycolysis. Most sites (91\%) use SUV to monitor treatment response, but only $35 \%$ of sites use SUV to discriminate malignant from benign uptake. Reporting times ranged from 5 to $90 \mathrm{~min}$, with $70 \%$ of sites requiring $30 \mathrm{~min}$ or less and only $7 \%$ taking longer than $1 \mathrm{~h}$.

After PET/CT data are processed and reported, they are archived. Users reported storage of CT DICOM images (93\%), PET DICOM images (98\%), CT raw data (12\%), PET raw data (26\%), user-defined screenshots $(50 \%)$, and PET maximum-intensity projections (57\%). At only $38 \%$ of sites are PET/CT images made available on a compact disk

TABLE 6

Percentage of Sites Using Various CT Parameters

Percentage of sites using this parameter in. .

\begin{tabular}{lcccc}
\hline & \multicolumn{3}{c}{ Percentage of sites using this parameter in... } \\
\cline { 2 - 5 } \multicolumn{1}{c}{ Protocol parameter } & No patients & Very few patients (1\%-25\%) & $\begin{array}{c}\text { Some patients } \\
(26 \%-75 \%)\end{array}$ & $\begin{array}{c}\text { Most patients } \\
(76 \%-100 \%)\end{array}$ \\
\hline Intravenous CT contrast material & 31 & 25 & 24 & 20 \\
Oral CT contrast material & 28 & 13 & 12 & 46 \\
Breathing instructions during CT & 44 & 8 & 5 & 44 \\
Respiratory gating & 70 & 18 & 3 & 0 \\
Furosemide & 73 & 20 & 0 & 6 \\
Bladder catheter & 80 & & & 0 \\
\hline
\end{tabular}


together with a DICOM viewer to patients and referring physicians.

\section{DISCUSSION}

The aim of this survey was to gather data on PET/CT practice and operational heterogeneity in clinical routine. The total response rate of $14 \%$ is within accepted response ranges for Web-based surveys $(9,10)$. Most responses were collected from users in the United States. Today, about $45 \%$ and $15 \%$ of PET/CT systems are installed in the United States and Europe, respectively, which corresponds roughly to the regional input collected in our survey. PET/CT operations in Asia-Pacific are reflected less adequately, based on the number of responses collected from that region. The overall fraction of responses from public (60\%) and private settings $(30 \%)$ corresponds roughly to the public and private operations of PET/CT in Europe (80\% and 20\%, respectively) and Germany ( $80 \%$ and $20 \%$, respectively).

In general, total numbers of PET/CT installations and operations are unknown, aside from estimates provided in recent publications on the status of PET in Germany (15), PET/CT in India (16), and trends in PET ownership in the United States (17).

According to our survey, only $24 \%$ of all PET/CT systems are operated at multidisciplinary centers, such as dedicated PET/CT centers, cancer centers, and diagnostic imaging centers, thus illustrating the close collaboration that is required among clinical and imaging specialists for meaningful ${ }^{18} \mathrm{~F}$-FDG PET/CT image interpretation despite an ongoing "ownership" debate $(7,18)$. Our survey found that the adoption of PET/CT appeared unrelated to experience with stand-alone PET systems: 59\% of sites had prior PET experience and $41 \%$ of sites did not. Most sites had 4, 5, or over $10 \mathrm{y}$ of PET-only experience before the installation of a PET/CT system.

The answers to questions about the average number of staff members involved in clinical PET/CT operations raise several points. First, it appears that many more technologists than physicists or medical doctors are involved. A possible reason may be the fact that some sites, governed by their local law, need to staff their PET/CT systems with both a radiology-trained technologist and a nuclear medicinetrained technologist. Second, systems with high throughput may require the availability of a larger number of technologists in order to limit the total radiation burden of each. Minimum physics support per PET/CT operation appears to have decreased since the onset of this technology, illustrating its more routine availability and greater use today. There appear to be more nuclear medicine experts involved than radiologists, as is reflected also in the low frequency of contrast-enhanced PET/CT examinations (Table 6). Only 2 sites reported the engagement of dual-board-certified physicians. This fraction may change as more residents welcome a combined radiology and nuclear medicine training program. A recent survey by the European Society of Radiology and EANM showed that $77 \%-84 \%$ of all members of those 2 societies favor an interdisciplinary training program (19).

Based on the survey results, the 3 main diseases studied with PET/CT are lymphoma, lung cancer, and breast cancer, followed by colon cancer and head/neck cancer. This response corresponds to most of the PET/CT indications identified in a recent study from Germany (15), although the ranking of lymphoma studies and lung studies is reversed. The 2 main indications for cardiac PET/CT were myocardial viability studies and rest-stress perfusion studies. PET/CT in neurology was used mostly for dementia workups, brain tumors, and epilepsy (Table 3).

Overall, PET/CT was used for oncology, radiation therapy planning, cardiology, and neurology in, respectively, $87 \%, 4 \%, 4 \%$ and $5 \%$ of patients. This response corresponds to the uses discussed by Kotzerke et al. (15).

An important factor in judging the availability of PET/ CT technology is the average time patients have to wait for an appointment. In some countries, national health boards have put forward recommendations on cancer patient workups, including recommendations on the choice of modality and reasonable waiting times (20). Our survey indicated that $9 \%-15 \%$ of patients had to wait more than 1 wk for a PET/CT appointment.

Optimal and appropriate use of PET/CT requires attention to the quality of CT and PET images. Adherence to the technical and clinical guidelines put forward by multidisciplinary expert panels (4-6) ensures a minimum set of quality standards, simplifies cross-center and cross-system data comparison, and is a basis for the standardization of PET/CT (21) in multicenter studies. Our survey included several additional questions about the PET/CT protocol (Table 6).

Although current guidelines suggest a minimum fasting period of 4-6 $\mathrm{h}$ before a ${ }^{18} \mathrm{~F}-\mathrm{FDG}$ PET examination, our survey indicated a striking variability in minimum fasting time: $72 \%$ of sites adhered to $4-6 \mathrm{~h}$, whereas $21 \%$ of sites extended the fasting period to up to $20 \mathrm{~h}$. One third of the sites established additional, frequently nonsensical, dietary requirements for oncology studies. Most sites (99\%) measure blood glucose levels before the administration of ${ }^{18} \mathrm{~F}-\mathrm{FDG}$. However, cutoff values varied from $120 \mathrm{mg} / \mathrm{dL}$ (6) to 150 $200 \mathrm{mg} / \mathrm{dL}(4,15)$. Interestingly, $3 \%$ of sites set their cutoff at $250 \mathrm{mg} / \mathrm{dL}$, whereas $7 \%$ use no cutoff (Supplemental Fig. 2).

Across all sites, the maximum ${ }^{18} \mathrm{~F}-\mathrm{FDG}$ activities injected were $465 \mathrm{MBq}$ and $524 \mathrm{MBq}$ for 3D PET and 2D PET, respectively and, thus, were within the recommended activity range of $360-740 \mathrm{MBq}$ (4). However, with novel PET detector materials and the availability of 3D and time-of-flight PET acquisitions, these activity ranges could be lowered without significant degradation of PET image quality (6). Accordingly, the EANM suggests using $380 \mathrm{MBq}$ and $190 \mathrm{MBq}$ for 2D PET and 3D PET, respectively, for a standard adult patient $(75 \mathrm{~kg})$. In obese patients, the maximum injected activity should be below $530 \mathrm{MBq}-$ far lower than the maximum activities found in our survey, which were $740 \mathrm{MBq}$ and $670 \mathrm{MBq}$ for 3D PET and 2D 
PET, respectively. In many patients, such activities lead to acquisitions beyond the peak noise-equivalent counts. Therefore, particularly for heavy patients, extended emission acquisitions may yield image quality superior to that after increased activity levels, as shown by Masuda et al. (22) and as recommended by the Dutch protocol for standardized whole-body ${ }^{18}$ F-FDG PET/CT (21) and by the EANM (6). Patient weight-adapted emission imaging, first suggested by Halpern et al. (23), was used by $44 \%$ of the surveyed sites.

Tracer uptake time varied widely in our survey, nor is any consensus apparent from the 3 major guidelines. All surveyed sites adhere to an ${ }^{18} \mathrm{~F}$-FDG uptake time of at least $45-$ $90 \mathrm{~min}$. Boellaard et al. limit the uptake time to $55-65 \mathrm{~min}$ (6), with which only $47 \%$ of the surveyed sites complied. Strict compliance with predefined uptake times is essential for high reproducibility within and among institutions and for comparability between serial studies. Likewise, a wide range of field-of-view limits was evident for torso PET/CT examinations. Unfortunately, existing guidelines are noncommittal. For example, Delbeke et al. defined a torso acquisition as one extending from "skull base to midthigh" (4), whereas Krause et al. defined the same examination range ("skull base to proximal thighs") as being "whole body" (5). Similarly, Boellaard et al. refer to the "base of the skull base to mid thigh" range as being "whole body" ( 6 ). The results of our survey uncovered this confusion because sites reported various anatomic landmarks and simplified descriptors of coaxial imaging limits (Fig. 1).

Most sites $(73 \%)$ use a dedicated low-dose CT scan for attenuation correction, consistent with guidelines aimed at minimizing patient radiation exposure. The use of contrast-enhanced CT images for attenuation correction is still a matter of debate $(24,25)$. A recent poll among radiologists and nuclear medicine physicians indicated that $60 \%$ of experts anticipated a growing use of contrastenhanced CT as part of integrated PET/CT (19).

Of the sites we surveyed, $31 \%$ used no intravenous contrast material, whereas $20 \%$ used intravenous contrast material in most patients. Oral CT contrast material was used in most patients by $46 \%$ of sites. This preference for oral over intravenous contrast material is striking because oral contrast material may yield artifactual uptake patterns and bias in attenuation-corrected PET, much like focal concentrations of intravenous contrast material (26), unless a water-based oral agent $(27,28)$ is used. However, only $26 \%$ of the respondents who use oral contrast agents use a water-based agent. Clearly, further training seems appropriate to help PET/CT users understand the potential bias introduced by CT contrast material and to leverage the full potential of diagnostic PET/CT.

Respiration artifacts are known to occur in PET/CT images if the CT images are acquired during a respiratory phase significantly different from mid expiration. Forty-four percent of our surveyed sites use breathing instructions (29) in most patients, whereas another $44 \%$ do not. Those sites that do not use breathing instructions may use PET/CT systems with multislice $(n>6)$ CT technology, in which quiet breathing would be acceptable (30). Alternatively, respiratory gating is available with state-of-the-art PET/CT technology and can also be used to improve CT-based attenuation correction (31). However, this approach is not popular and was used by only $20 \%$ of the sites. Similarly, the use of furosemide, a bladder catheter, or anesthesia is an option to further improve PET/CT image quality but, because of the invasiveness and associated patient discomfort, remains unpopular (Table 6). Other efforts toward increasing diagnostic quality may include dedicated positioning devices that help immobilize the patient comfortably for the duration of the examination $(32,33) ; 41 \%$ of sites use such devices.

PET image reconstruction differed among the sites: $48 \%$ appeared to apply standard, predefined reconstruction protocols on $128 \times 128$ matrices only. However, higherresolution PET images are feasible and beneficial when one is imaging smaller objects, such as the head, neck, or extremities. Therefore, indication-specific adjustments to reconstruction parameters have been recommended $(6,21)$. Such suggestions will help to standardize PET image acquisitions and analyses across institutions.

Further, an initial choice of optimum reconstruction parameters will help minimize repeated reconstructions, for which access to the raw emission sinogram data, at least, needs to be ensured. Only $22 \%$ of the sites store the emission sinograms. A considerable number of sites still report PET and CT separately; only $63 \%$ of the clinical reports are fully integrated, whereas for $17 \%$ of patients 2 separate reports are generated. Reports on combined PET/ CT examinations should be integrated (4) not only to justify the use of a dual-modality technique but perhaps more so to inform the referring physicians adequately and not leave them with a decision to make based on potentially differing $\mathrm{CT}$ and PET reports.

Clearly, joint reading of PET/CT studies requires advanced training on the dual-modality technique, and various suggestions have been made to address this topic (19). Practical approaches to bring about dual-modality training need to be developed. Alternatively, dual-certified physicians could issue PET/CT reports. Our survey indicated that $37 \%$ of PET/CT reports are issued by dual-certified physicians whereas only $23 \%$ are issued by more than one physician jointly; all other reports are issued by either radiologists or nuclear medicine physicians.

Variations in the reported SUV parameter do exist; some of these variations result from the inability to automatically calculate total lesion glycolysis - or SUV corrected for lean body mass or body surface area-with existing image analysis platforms. Nonetheless, available SUV measurements should be included in the report, but only $90 \%$ of sites did so.

The time required for interpretation was as long as the time of actual image acquisition. With an average emission scan time of $3 \mathrm{~min}$ per bed position, a torso scan is completed in less than $25 \mathrm{~min}$ assuming a 7-bed-position 
examination. At our surveyed sites, $51 \%$ of PET/CT reports take 16-30 min, raising concern about the quality of reports in high-throughput scenarios with only limited staff available. Finally, it would seem appropriate to provide referring physicians with a copy of the PET/CT image data together with the report. However, only $30 \%$ of sites indicated that they do so for most patients.

This study had some limitations. First, we collected data on the heterogeneity of PET/CT operations in clinical practice. Because those who respond to a survey such as ours are typically the experts who are most interested and motivated (34), we assume that the actual operational and procedural heterogeneities are far larger than reported here. Second, several responders did not provide eligible answers to every question asked (so-called item nonresponse (35)). A problem inherent to survey research itself, regardless of format or mode of administration, is that maximizing the breadth of a survey increases the risk of a greater number of incomplete responses. Therefore, we could not categorize responses to questions on PET/CT protocols with respect to the origin of the response (e.g., public vs. private settings or United States vs. Asia-Pacific). Finally, our survey did not address in detail all aspects of PET/CT acquisition protocols. We composed a series of questions that - based on our experience-mirror the areas of greatest diversity among clinical PET/CT users. If adherence to guidelines were to be tested in clinical reality, a regional or global survey performed by a societal organization would be best. In addition, it seems mandatory that radiology and nuclear medicine associations need to work together on a global scale to clarify the existing ambiguities between guidelines.

\section{CONCLUSION}

Our survey revealed significant variations in the way standard ${ }^{18} \mathrm{~F}-\mathrm{FDG}$ PET/CT acquisitions are conducted among clinical PET/CT users worldwide. Severe and frequent deviations from recommended guidelines are observed in such areas as patient preparation, injected activities, and imaging preconditions. Variability in imaging acquisition parameters indicates that standardization can be improved. Continuous training and international efforts at further standardization are urgently needed to optimize the use of PET/CT in oncology.

\section{ACKNOWLEDGMENTS}

We thank the Academy of Molecular Imaging for endorsing and supporting this survey. We are grateful to Nichole Anvar, in particular, for her support in setting up the survey and gathering the responses. Thomas Beyer is the chief executive officer of Switzerland-based cmi-experts $\mathrm{GmbH}$. Johannes Czernin is a cofounder of Momentum Biosciences and Sofie Biosciences, Los Angeles, California, and serves as an advisor to cmi-experts GmbH. Lutz S. Freudenberg serves as an advisor to cmi-experts $\mathrm{GmbH}$ and has received speaker fees from Siemens Healthcare, Philips Healthcare, and Genzyme Corp.

\section{REFERENCES}

1. Townsend DW. Multimodality imaging of structure and function. Phys Med Biol. 2008;53:R1-R39.

2. Czernin J, Allen-Auerbach M, Schelbert H. Improvements in cancer staging with PET/CT: literature-based evidence as of September 2006. J Nucl Med. 2007;48 (suppl 1):78S-88S.

3. Shankar LK, Hoffmann JM, Bacharach S, et al. Consensus recommendations for the use of ${ }^{18} \mathrm{~F}$-FDG PET as an indicator of therapeutic response in patients in national Cancer Institute Trials. J Nucl Med. 2006;47:1059-1066.

4. Delbeke D, Coleman RE, Guiberteau MJ, et al. Procedure guideline for tumor imaging with ${ }^{18}$ F-FDG PET/CT 1.0. J Nucl Med. 2006;47:885-895.

5. Krause BJ, Beyer T, Bockisch A, et al. FDG-PET/CT in oncology: German guideline [in German]. Nuklearmedizin. 2007;46:291-301.

6. Boellaard R, O'Doherty MJ, Weber WA, et al. FDG PET and PET/CT: EANM procedure guidelines for tumour PET imaging: version 1.0. Eur J Nucl Med Mol Imaging. 2010;37:181-200.

7. Bischof Delaloye A, Carrió I, Cuocolo A, et al. White paper of the European Association of Nuclear Medicine (EANM) and the European Society of Radiology (ESR) on multimodality imaging. Eur J Nucl Med Mol Imaging. 2007; 34:1147-1151

8. Graham MM, Badawi RD, Wahl RL. Variations in PET/CT methodology for oncologic imaging at U.S. academic medical centers: an Imaging Response Assessment Team survey. J Nucl Med. 2001;51:311-317

9. Manfreda KL, Bosnjak M, Berzelak J, et al. Web surveys versus other survey modes: a meta-analysis comparing response rates. Int J Market Res. 2008;50:79-104.

10. Shih T, Fan X. Comparing response rates from web and mail surveys: a metaanalysis. Field Methods. 2008;20:249-271.

11. Cook C, Heath F, Thompson RA. Meta-analysis of response rates in Web- or Internet-based surveys. Educ Psychol Meas. 2000;60:821-838.

12. Deutskens E, de Ruyter K, Wetzels M, Oosterveld P. Response rate and response quality of Internet-based surveys: an experimental study. Mark Lett. 2004;15:21-36.

13. Edwards PJ, Roberts I, Clarke MJ, et al. Methods to increase response to postal and electronic questionnaires. Cochrane Database Syst Rev. 2009;(3): MR000008.

14. Beyer T, Antoch G, Mueller S, et al. Acquisition protocol considerations for combined PET/CT imaging. J Nucl Med. 2004;45(suppl 1):25S-35S.

15. Kotzerke J, Oehme L, Lindner O, et al. Positron emission tomography 2008 in Germany: results of the query and current status. Nuklearmedizin. 2010;49:58-64.

16. Rangarajan V, Purandare NC, Sharma AR, Shah S. PET/CT: current status in India. Indian J Radiol Imaging. 2008;18:290-294.

17. Agarwal R, Levin DC, Parker L, Rao VM. Trends in PET scanner ownership and leasing by nonradiologist physicians. J Am Coll Radiol. 2010;7:187-191.

18. Biersack HJ. PET-CT: comments on the white paper [letter]. Eur J Nucl Med Mol Imaging. 2008;35:1576.

19. Cuocolo A, Breatnach E. Multimodality imaging in Europe: a survey by the European Association of Nuclear Medicine (EANM) and the European Society of Radiology (ESR). Eur J Nucl Med Mol Imaging. 2010;37:163-167.

20. Denmark National Board of Health Web site. Cancer Pathways page. Available at: http://www.sst.dk/English/cancer\%20pathways.aspx?sc_lang=en. Accessed September 2. 2010.

21. Boellaard R, Oyen WJ, Hoekstra CJ, et al. The Netherlands protocol for standardisation and quantification of FDG whole body PET studies in multi-centre trials. Eur J Nucl Med Mol Imaging. 2008;35:2320-2333.

22. Masuda Y, Kondo C, Matuso Y, et al. Comparison of imaging protocols for ${ }^{18} \mathrm{~F}$ FDG PET/CT in overweight patients: optimizing scan duration versus administered dose. J Nucl Med. 2009;50:844-848.

23. Halpern BS, Dahlbom M, Auerbach MA, et al. Optimizing imaging protocols for overweight and obese patients: a lutetium orthosilicate PET/CT study. $\mathrm{J}$ Nucl Med. 2005;46:603-607.

24. Kuehl H, Antoch G. How much CT do we need for PET/CT: a radiologist's perspective. Nuklearmedizin. 2005;44(suppl):S24-S31.

25. Kuehl H, Veit P, Rosenbaum SJ, et al. Can PET/CT replace separate diagnostic $\mathrm{CT}$ for cancer imaging? Optimizing CT protocols for imaging cancers of the chest and abdomen. J Nucl Med. 2007;48(suppl 1):45S-57S.

26. Kinahan P, Hasegawa B, Beyer T. X-ray based attenuation correction for positron emission tomography/computed tomography scanners. Semin Nucl Med. 2003; 33:166-179.

27. Antoch G, Kuehl H, Kanja J, et al. Dual-modality PET/CT scanning with negative oral contrast agent to avoid artifacts: introduction and evaluation. Radiology. 2004;230:879-885. 
28. Hausegger $\mathrm{K}$, Reinprecht $\mathrm{P}$, Kau T, et al. Clinical experience with a commercially available negative oral contrast medium in PET/CT. Rofo. 2005;177:796-799.

29. Beyer T, Antoch G, Blodgett T, et al. Dual-modality PET/CT imaging: the effect of respiratory motion on combined image quality in clinical oncology. Eur $J$ Nucl Med Mol Imaging. 2003;30:588-596.

30. Beyer T, Rosenbaum S, Veit $\mathrm{P}$, et al. Respiration artifacts in whole-body ${ }^{18} \mathrm{~F}-$ FDG PET/CT studies with combined PET/CT tomographs employing spiral CT technology with 1 to 16 detector rows. Eur J Nucl Med Mol Imaging. 2005;32: 1429-1439.

31. Büther F, Dawood M, Stegger L, et al. List mode-driven cardiac and respiratory gating in PET. J Nucl Med. 2009;59:674-681.
32. Heusner TA, Kuemmel S, Hahn S, et al. Diagnostic value of full-dose FDG PET/ CT for axillary lymph node staging in breast cancer patients. Eur J Nucl Med Mol Imaging. 2009;36:1543-1550.

33. Brechtel K, Heners H, Mueller M, et al. Fixation devices for whole-body ${ }^{18} \mathrm{~F}-$ FDG PET/CT: patient perspectives and technical aspects. Nucl Med Commun. 2007;28:141-147.

34. Sax LJ, Gilmartin SK, Bryant AN. Assessing response rates and nonresponse bias in Web and paper surveys. Res Higher Educ. 2003;44: 409-432.

35. Fraenkel JR, Wallen NE. How to Design and Evaluate Research in Education. 6th ed. New York, NY: McGraw Hill; 2005;424:210-211. 